\title{
Dolor neuropático en pacientes diabéticos insulinodependientes
}

\section{Neuropathic pain in insulin-dependent diabetic patients}

\author{
Prof. Dra. Yaquelín Pérez-Guirola,* Dr. Adrián Lombas-Rojas, ${ }^{\ddagger}$ \\ Prof. Dra. Cs. Idoris Cordero-Escobar ${ }^{\S}$
}

Citar como: Pérez-Guirola Y, Lombas-Rojas A, Cordero-Escobar I. Dolor neuropático en pacientes diabéticos insulinodependientes. Rev Mex Anest. 2021; 44 (1): 51-54. https://dx.doi.org/10.35366/97777

RESUMEN. Introducción: El dolor neuropático es complejo y, a pesar de los avances farmacológicos y no farmacológicos de los últimos años, es la principal causa de sufrimiento en pacientes diabéticos insulinodependientes. Objetivo: Realizar una revisión no sistemática de la literatura sobre el dolor neuropático en el paciente diabético insulinodependiente. Material y métodos: Se realizó una búsqueda bibliográfica de la literatura, el día 03 de agosto de 2019, en las bases de datos PubMed, de la Biblioteca Médica Nacional de los Estados Unidos (NLM), con los siguientes descriptores en idioma inglés: diabetes mellitus insulinodependiente AND neuropatic pain, lo cual ofreció un total de 133 publicaciones. Cuando se activó el filtro en humanos se mantuvieron 11 artículos. Con el filtro en los últimos cinco años, a texto completo, permaneció uno cuyo título no está relacionado con el proyecto propuesto. Conclusiones: El paciente diabético insulinodependiente, en sentido general, presenta dolor neuropático de intensidad variable, que afecta su calidad de vida y que requiere tratamiento especializado.

ABSTRACT. Introduction: Neuropathic pain is complex and despite the pharmacological and non-pharmacological advances of recent years, it is the main cause of suffering in insulin-dependent diabetic patients. Objective: To update a non-systematic review of the literature on neuropathic pain in the insulin-dependent diabetic patient. Material and methods: A literature search was conducted on August 3 of 2019 in the PubMed databases of the National Medical Library of the United States (NLM), with the following English-language descriptors: insulin-dependent diabetes mellitus AND neuropathic pain, which offered a total of 133 publications. When the filter was activated in humans, 11 articles were maintained. With the filter in the last five years, full text remained one whose title is not related to the proposed project. Conclusions: The general insulin-dependent diabetic patient presents with neuropathic pain of varying intensity, which affects their quality of life and requires specialized treatment.

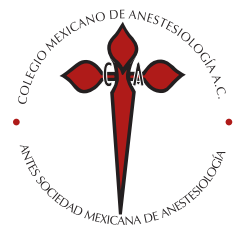

Palabras clave:

Neuropatías diabéticas, diabetes mellitus, dolor neuropático.

Keywords:

Diabetic neuropathies, diabetes mellitus, neuropathic pain.

\begin{abstract}
* Especialista en Anestesiología
y Reanimación. Profesora

Auxiliar. Máster en Urgencias

Médicas. Clínica del Dolor. ORCID. 0000000309082287.

‡ Especialista en Anestesiología y Reanimación. ORCID. http:// orcid.org/0000-0002-2912-6227.

$\S$ Especialista en Anestesiología y Reanimación. Profesora e Investigadora Titular. ORCID: http:// orcid.org/0000-0001-9877-3113.
\end{abstract}

Hospital Clínico Quirúrgico «Hermanos Ameijeiras». La Habana, Cuba.

Correspondencia:

Prof. Dra. Cs. Idoris

Cordero Escobar

E-mail: ice@infomed.sld.cu

Recibido para publicación: 19-09-2019

Aceptado para publicación: 04-02-2020

\section{INTRODUCCIÓN}

$\mathrm{E}$ l dolor de origen neuropático es muy complejo. La variación en la presentación de los síntomas y la falta de estudios diagnósticos precisos, en muchos casos, inducen un mal tratamiento del paciente ${ }^{(1)}$.

Del total de los pacientes tratados muchos experimentan mejoría con diversos tratamientos; pero hay un número considerable de ellos que permanecen discapacitados ${ }^{(2)}$.

En los últimos años se ha logrado comprender más y mejor los mecanismos subyacentes de estos síndromes, que tienen un denominador común: el dolor. Sin lugar a dudas, este hecho va a contribuir al desarrollo de estrategias más efectivas para su tratamiento ${ }^{(3)}$.
El dolor neuropático (DN) es una de las complicaciones frecuentes de la diabetes mellitus (DM) y se produce como consecuencia de la disfunción de los nervios periféricos. Se manifiesta, en la mayoría de los pacientes, por aparición de dolor de tipo específico severo, sufrimiento, altos grados de invalidez e importante deterioro de la calidad de vida ${ }^{(4)}$.

Este tipo de dolor se percibe como el resultado de la integración de dos sensaciones: la dimensión física o sensorial y la psíquica. Éstas influyen significativamente en la esfera emocional, la cual interviene en la ansiedad o en la depresión que enrarecen la percepción sensorial y que aportan elementos de amplificación que suelen complicar la terapia cuando el dolor permanece durante largo tiempo $^{(5-10)}$. 
La DM puede ocasionar neuropatías a diferentes niveles, por lo que el estudio y la valoración de las dimensiones del dolor en cada enfermo con DN no es una cuestión semántica, sino que tiene un interés terapéutico indudable $\mathrm{e}^{(11-15)}$.

Constituye el objetivo de este artículo realizar una revisión de la literatura actualizada sobre el dolor neuropático en pacientes diabéticos insulinodependientes.

\section{MATERIAL Y MÉTODOS}

Diseño metodológico. Se realizó una búsqueda bibliográfica de la literatura, el día 03 de agosto de 2019, en las bases de datos PubMed, de la Biblioteca Médica Nacional de los Estados Unidos (NLM), con los siguientes descriptores en idioma inglés: diabetes mellitus insulinodependiente AND neuropathic pain, la que ofreció un total de 133 publicaciones. Cuando se activó el filtro en humanos se mantuvieron 11 artículos. Con el filtro en los últimos cinco años, a texto completo, permaneció uno cuyo título no está relacionado con el proyecto propuesto ${ }^{(11)}$.

\section{Desarrollo}

La redefinición del dolor neuropático como «dolor que surge como consecuencia directa de una lesión o enfermedad que afecta el sistema somatosensorial», el cual fue sugerido por la Asociación Internacional para el Estudio del Dolor (IASP) y del Grupo de Interés Especial sobre Dolor Neuropático (NeuPSIG) en 2008, se encuentra ampliamente aceptado ${ }^{(16)}$.

Existen en la piel nociceptores polimodales (responden a estímulos mecánicos, térmicos y químicos) y nociceptores silentes (aquéllos que se activan sólo cuando hay un proceso inflamatorio) $)^{(16)}$. En los tejidos profundos sólo hay nociceptores silentes. Los nociceptores están asociados con axones mielínicos delgados $\mathrm{A} \delta(\sim 4 \mu$ de diámetro, de conducción $\sim 15 \mathrm{~m} / \mathrm{s})$ y fundamentalmente con axones amielínicos $(<2$ $\mathrm{m} / \mathrm{s}$ de conducción mucho más lenta) que transmiten la señal nociceptiva hacia el sistema nervioso central (SNC) ${ }^{(17)}$.

A la entrada del asta posterior de la médula, las aferencias nociceptivas hacen sinapsis con neuronas nociceptivas específicas que proyectan sus axones por la vía espinotalámica contralateral al cerebro. Existen también en el asta posterior (láminas II y V de Rexed), neuronas de proyección de la vía espinotalámica en las que convergen aferencias nociceptivas así como de sensaciones táctiles y térmicas inocuas. Estas neuronas se han denominado WDR (Wide Dynamic Range o de rango dinámico amplio) y serían las responsables de síntomas como el dolor evocado por un estímulo normalmente no doloroso, también conocido como alodinia ${ }^{(18)}$.

Las proyecciones desde la vía espinotalámica hacen sinapsis en núcleos talámicos desde donde proyectan sus referencias hacia la corteza, que procesaría el dolor en el área sensitiva secundaria en la porción posterior de la ínsula. Paralelamente la aferencia nociceptiva se proyecta a la corteza del cíngulo anterior y corteza prefrontal ${ }^{(19)}$.

Tratar el dolor en estos pacientes es imprescindible. Éste representa un verdadero desafío tanto diagnóstico como terapéutico. La dificultad diagnóstica se origina principalmente en la presentación del cuadro clínico. Sin duda que un diagnóstico preciso permitirá la elección de estrategias adecuadas y resultará en un mayor porcentaje de éxito al tratamiento ${ }^{(20-25)}$.

Maier y colaboradores ${ }^{(7)}$ evaluaron a 1,236 pacientes con un diagnóstico clínico de DN mediante pruebas sensoriales cuantitativas (QST), de acuerdo con el protocolo de DFNS (Red Alemana de Investigación sobre Dolor Neuropático). Utilizaron estímulos nociceptivos térmicos y mecánicos, así como no estimuladores nociceptivos.

El sistema nociceptivo está adaptado para activarse con señales que son capaces de generar daño hístico. Por este motivo, los umbrales de activación de la vía nociceptiva fueron bajos. En el aspecto mecánico el roce de la piel, las temperaturas de $39^{\circ} \mathrm{C}$ o más, o temperaturas de $20^{\circ} \mathrm{C}$ o menos son capaces de generar potenciales de acción en aferentes nociceptivos para explorar el espectro de anormalidades sensoriales.

El no reconocer esta diferencia de temperatura es la que genera una serie de dificultades en el diagnóstico en pacientes con síndromes dolorosos neuropáticos ${ }^{(20-26)}$.

Bases fisiopatológicas para el tratamiento. El estrés oxidativo es un factor determinante en la patogénesis del DN, por lo que es prioritario el control de la hiperglucemia. La fuerte asociación entre la hiperglucemia, desarrollo y severidad del DN se demuestra en su prevalencia en los pacientes con un control glucémico insuficiente. La meta para el control glucémico recomendada debe ser, generalmente, menos de $7 \%$ de $\mathrm{HbA} 1 \mathrm{c}$, aunque se debe individualizar de acuerdo al perfil clínico del paciente. Hasta 30\% de los pacientes con DM1 desarrollan neuropatías diabéticas y $\mathrm{DN}^{(14)}$.

\section{Tratamiento del dolor neuropático(14)}

Antidepresivos tricíclicos (ADT). Se recomiendan amitriptilina e imipramina. La primera se indica como dosis de inicio a partir de los $10 \mathrm{mg}$. La prescripción en dosis nocturnas es debido a que puede ocasionar somnolencia. Los fármacos tricíclicos se han utilizado desde hace más de 30 años en el tratamiento del dolor neuropático del diabético. Su mecanismo de acción es la sinapsis neuronal a nivel de las vías descendentes del SNC y su modo de acción es la inhibición de la absorción de noradrenalina y serotonina, además de su acción como bloqueante de los receptores $\mathrm{N}$-metil-aspartato, que actúa como mediador de la hiperalgesia y la alodinia. Su dosis media suele ser de $10 \mathrm{mg}$, es el fármaco de elección entre los ADT. Presenta frecuentes efectos adversos secundarios de tipo colinérgico, sobre todo en pacientes mayores de 60 años. Está contraindicada en casos 
de glaucoma, hiperplasia prostática o arritmias cardíacas. Se debe tener presente su efecto de hiperorexia en los pacientes con obesidad y/o síndromes plurimetabólicos.

Estas recomendaciones se sustentan en que la mayoría de los estudios de clase 1 y 2 se han realizado con la amitriptilina, es un tratamiento de larga duración y de menos costo que la imipramina. Estas indicaciones están avaladas por estudios de Cochrane y otros metaanálisis. De acuerdo con el Grupo Operativo Europeo (European Task Force), dedicado al tratamiento del DN para este grupo de fármacos, es recomendado como tratamiento de primera línea, nivel de evidencia A (NE A), recomendación I ${ }^{(14,15)}$.

Inhibidores de la absorción de recaptación de serotonina y noradrenalina ${ }^{(16-22)}$. La eficacia de la duloxetina se ha avalado por múltiples estudios aleatorizados que demuestran que las dosis de 60 a $120 \mathrm{mg}$ responden mejor a las algias agudas que en las de carácter penetrante, urente e intenso, con bajo abandono del tratamiento debido a la buena tolerancia (20\%).

Se recomienda su empleo a dosis iniciales de $30 \mathrm{mg}$ (NE A, recomendación I).

\section{Fármacos anticonvulsivantes ${ }^{(23-26)}$}

Carbamazepina. Los efectos tóxicos limitan su uso. No se recomienda como tratamiento de primera línea. (NE B, recomendación III).

Fenitoína (difenilhidantoína). Es una opción para el dolor neuropático en el servicio de urgencias, dosis intravenosa de $15 \mathrm{mg} / \mathrm{kg}$. Se debe considerar efectos adversos. (NE C, recomendación IIb).

Gabapentina. Dosis: 900-3,600 mg/día (oral). Es una elección cuando los ADT no son tolerados. Actúa sobre los receptores GABA de las fibras nerviosas a nivel central. No se recomienda como tratamiento de primera línea debido a evidencia limitada. (NE B, recomendación III) ${ }^{(28)}$.

Pregabalina. Se trata de un análogo de la gabapentina que actúa sobre los receptores GABA a nivel del sistema nervioso central. Produce bloqueo de los canales iónicos. Una dosis inicial de 150-600 mg/día, repartida en dos veces, logra una reducción del dolor de hasta 50\%. Aparece mejoría desde la primera a la quinta semana de tratamiento. Los efectos adversos son somnolencia, edemas, mareos y náuseas. Se recomienda como fármaco de primera línea. Tiene concedida la autorización para el tratamiento del dolor en EUA y la Unión Europea. (NE A, recomendación I) ${ }^{(27)}$.

Antiarrítmicos. Lidocaína. Anestésico local, de escasa tolerancia y duración limitada respecto a la mexiletina. La dosis de 675 mg/día es de acción rápida.

No se recomienda como tratamiento de primera línea. (NE C, recomendación II) ${ }^{(28)}$.

Analgésicos. Los antiinflamatorios no esteroideos (AINEs) rara vez son útiles en el tratamiento y no se recomienda su empleo. (NE C, recomendación III) ${ }^{(9)}$.
Tramadol. Es un opioide sintético. Actúa sobre los receptores opioides, así como sobre los monoaminérgicos. Las dosis recomendadas son de 50 a $400 \mathrm{mg} /$ día (oral). No se recomienda como tratamiento de primera línea; puede combinarse con otros fármacos (antidepresivos o anticonvulsivantes) como fármaco de segunda línea en situaciones clínicas de rescate. (NE B, recomendación Ia: como tratamiento de segunda línea ante sólo necesidad de terapia de rescate temporal) ${ }^{(9)}$.

El tramadol, además de su actividad como agonista opioide débil, inhibe la recaptación de serotonina y noradrenalina. En la revisión sistemática del grupo NeuPSIG se halla evidencia de calidad moderada sobre su eficacia en el tratamiento del DN, pero debido a su menor tolerabilidad y seguridad se da una recomendación débil para su uso como segunda línea de tratamiento(26-29).

Oxicodona. La dosis usada habitualmente es de 10 a 60 mg/día. El potencial de dependencia de este opioide sintético de acción prolongada limita su uso en esta enfermedad. Sólo se debería emplear como tratamiento de rescate en situaciones extremas de dolor y teniendo presente todas las consecuencias de dependencia y las complicaciones del fármaco. (NE B, recomendación IIa) ${ }^{(27-29)}$.

En cuanto a los opioides mayores (morfina y oxicodona) son de los que más estudios se disponen. El NeuPSIG, con un grado de recomendación débil, les otorga lugar como tercera línea de tratamiento, principalmente debido a sus problemas de seguridad ${ }^{(27-29)}$. Sin embargo, la Guía NICE recomienda no utilizar la morfina fuera del ámbito especializado y en su actualización bibliográfica de 2017 no encuentra evidencia suficiente para propiciar una actualización de sus recomendaciones ${ }^{(22-25)}$.

En la Tabla 1 se resumen las dosis de fármacos a utilizar, las dosis iniciales y máximas recomendadas. Se debe considerar indicaciones, contraindicaciones, tolerancia y compatibilidad con el resto de fármacos ${ }^{(26-32)}$. Estos fármacos fueron aprobados por la Agencia Europea de Medicamentos (EMEA) y la Food and Drugs Agency (FDA) de los Estados Unidos para el tratamiento del dolor neuropático en pacientes diabéticos. Por otra parte, no existe evidencia de que la

\begin{tabular}{|c|c|c|c|}
\hline & Fármaco & $\begin{array}{c}\text { Dosis inicial } \\
\text { (mg/día) }\end{array}$ & $\begin{array}{c}\text { Dosis máxima } \\
\text { recomendada } \\
\text { (mg/día) }\end{array}$ \\
\hline \multirow[t]{2}{*}{ Primera línea } & Pregabalina & 100 & 300 \\
\hline & Duloxetina & 60 & 120 \\
\hline \multirow[t]{2}{*}{ Alternativos } & Amitriptilina & 25 & 150 \\
\hline & Gabapentina & 300 & 1,800 \\
\hline \multirow[t]{4}{*}{ Segunda línea } & Opioides & 50 & 200 \\
\hline & Tramadol & 10 & 60 \\
\hline & Oxicodona & Tópica & Tópica \\
\hline & Capsaicina & & \\
\hline
\end{tabular}


asociación entre la pregabalina y la duloxetina (grado A) sea superior al ajuste de la dosis individual. La gabapentina puede ser útil de inicio. Se debe tener en cuenta las comorbilidades e interacciones medicamentosas (grado B). Los antidepresivos tricíclicos (no aprobados por FDA) pueden ser efectivos con más efectos secundarios (grado B). Los opioides no se recomiendan como primera línea por sus efectos secundarios y su riesgo de adicción (grado D) ${ }^{(32,33)}$.
Se concluye que el paciente diabético insulinodependiente, en sentido general, presenta dolor neuropático de intensidad variable, que afecta su calidad de vida y que requiere tratamiento especializado.

Conflicto de intereses: Los autores declaran no tener conflicto de intereses.

\section{REFERENCIAS}

1. Pérez C, Ribera MV, Gálvez R, Micó JA, Barutell C, Failde I, et al. High prevalence of confirmed, butalsoof potential and believed, neuropathic pain in pain clinics. Eur J Pain. 2013;17:347-356.

2. Samper BD, Monerris TM, Homs RM, Soler PM. Etiología y manejo de la neuropatía diabética dolorosa. Rev Soc Esp Dolor. 2010;17:286298.

3. Flórez S, León M, Torres M, Reyes F, Serpa JC, Ríos AM. Manejo farmacológico del dolor neuropático. Rev Col Anest. 2009;37:356372.

4. Pérez C, Ribera MV, Gálvez R, Micó JA, Barutell C, Failde I, et al. High prevalence of confirmed, but also of potential and believed, neuropathic pain in pain clinics. Eur J Pain. 2013;17:347-356.

5. Camperos SM. Revista médica. Clínica Las Condes. 2014;25:602-609.

6. Declaration of Montreal. Declaration that access to pain management is a fundamental human right. [On line]. Consulted: June 2019. URL Available in: http://www.iasp-pain.org/DeclarationofMontreal?navItem Number $=582$.

7. Maier C, Baron R, Tölle TR, Binder A, Birbaumer N, Birklein F, et al. Quantitative somatosensory abnormalities in 1,236 patients with different neuropathic pain syndromes. Pain. 2010;150:384-385.

8. Gaspar AT, Antunes F. Type 1 complex regional pain syndrome. Acta Med Port. 2011;24:1031-1040.

9. Finnerup NB, Haroutounian S, Kamerman P, Baron R, Bennett DL, Bouhassira D, et al. Neuropathic pain: an updated grading system for research and clinical practice. Pain. 2016;157(8):1599-606.

10. Escaño-Polanco FM, Odriozola A, Davidson J, Pedrosa H, Fuente G, Márquez G, et al. Consenso de expertos para el manejo de la neuropatía diabética. Rev ALAD. 2016;6:121-150.

11. Moriyama Y, Hiasa M, Sakamoto S, Omote H, Nomura M. Vesicular nucleotide transporter (VNUT): appearance of an actress on the stage of purinergic signaling. Purinergic Signal. 2017;13:387-404.

12. Spallone V. Management of painful diabetic neuropathy: guideline guidance or jungle? Curr Diab Rep. 2012;12:403-413.

13. Capmourteres EM, Finkel D. Psychotropic coadjuvants in pain treatment. Vertex. 2002;48:100-110.

14. Zarei M, Sabetkasaei M, Moini Zanjani T. Paroxetine attenuates the development and existing pain in a rat model of neurophatic pain. Iran Biomed J. 2014;18:94-100.

15. Mick G, Baron R, Finnerup NB. What is localized neuropathic pain? A first proposal to characterize and define a widely used term. Pain Manage. 2012;1:71-77.

16. Haanpaa M, Treede R.D. Diagnosis and classification of neuropathic pain. IASP Pain Clinical Updates. 2010;18: [On Line]. Consulted. 15 October of 2019. URL. https://www.iasp-pain.org/PublicationsNews/ NewsletterIssue.aspx?ItemNumber=2083.

17. Saig G. Diabetic neuropathy. Handb Clin Neurol. 2013;115:579-589.

18. González-Escalada JR, Rodríguez MJ, Camba MA. Recomendaciones para el tratamiento del dolor neuropático. Rev Soc Esp Dolor. 2009;16:445-467.

19. Baron R. Neuropathic pain: a clinical perspective. Handb Exp Pharmacol. 2009;194:3-30.
20. Brown MJ, Bird SJ, Watling S, Kaleta H, Hayes L, Eckert S, et al. Zenarest study. Natural progression of diabetic peripheral neuropathy in the Zenarestat study population. Diabetes Care. 2009;27:1153-1159.

21. Schiavon F, Circhetta C, Dani L. The diabetic hand. Rheumatism. 2010;56:139-142

22. Mota M, Panus C, Mota E, Sfredel V, Patrascu A, Vanghelie L, et al. Hand abnormalities of the patients with diabetes mellitus. Rom J Intern Med. 2011;38-39:89-95.

23. Rodríguez JM, Menéndez JR, Trujillo Y. Radicales libres en la biomedicina y estrés oxidativo. Rev Cub Med Milit. 2011;30:15-20.

24. Pop-Busui R, Boulton JA, Feldman EL, Bril V, Freeman R, Malik RA, et al. Diabetic Neuropathy: a position statement by the American Diabetes Association. Diabetes Care. 2017;40:136-154.

25. American Diabetes Association. Microvascular complications and foot care. Diabetes Care. 2017;40:588-598.

26. Tesfaye S, Vileikyte L, Rayman G, Sindrup SH, Perkins BA, Baconja M, et al. Painful diabetic peripheral neuropathy: consensus recommendations on diagnosis, assessment and management. Diabetes Metab Res Rev. 2011;27:629-638.

27. De Dios del Valle R. González Torrejón M, Sacristán Rodea A. Manejo del dolor neuropático: la gabapentina como opción. Disponible en: http://scielo.isciii.es/scielo.php?script=sci_arttext\&pid $=$ S1131-57682001000400006.

28. Dy SM, Bennett WL, Sharma R, Zhang A, Waldfogel JM, Nesbit SA, et al. Complications and Treating Symptoms of Diabetic Peripheral Neuropathy. Comparative Effectiveness Review No. 187. (Prepared by the Johns Hopkins University Evidence-based Practice Center under Contract No. 290-2015-00006-I.) AHRQ Publication No. 17-EHC005-EF. Rockville, MD: Agency for Healthcare Research and Quality; March 2017. [On line] Consulted: 30 October of 2019. URL: https:/www.ncbi.nlm.nih.gov/books/NBK442335/pdf/Bookshelf_ NBK442335.pd.

29. Wiffen PJ, Derry S, Bell RF, Rice ASC, Tölle T, Phillips T, et al. Gabapentin for chronic neuropathic pain in adults. Cochrane Database of Systematic Reviews. 2017;6:CD007938.

30. Derry S, Wiffen PJ, Moore R, Quinlan J. Topical lidocaine for neuropathic pain in adults. Cochrane Database of Systematic Reviews. 2014;7:CD010958.

31. Oliveros-Lijap L, Ávila-Espinoza P, Ulloa V, Bernabe-Ortiz A. Calidad de vida en pacientes con neuropatía diabética periférica: estudio transversal en Lima, Perú. Acta Med Peru. 2018;35(3):160-7

32. FDA. Food and drugs administration. [On line] Consulted: 23 November of 2019. URL. https://www.registrarcorp. com/fda-facility-registration/?utm_source=google \&utm_ medium = c p c \& u t m_t e r m = +fda + + a g e n c y \& u t m _ content $=1054397892 \&$ utm_ campaign $=39400842 \&$ matchtype $=\bar{b}$ \&device $=$ \& \& gclid $=$ EAIaIQobChMIk4OfuZHA5 wIVVoNaBR0L6 AmIEAAYASAAEgKVhPD_BwE\&step $=1$.

33. Agencia Española de Medicamentos. [En línea] Consultado: 23 Noviembre de 2019. Disponible en: https://www.aemps.gob.es/informa/ alertas/home.htm. 